\title{
An Efficient Detection of Moroccan Coastal Upwelling Based on Fusion of Chlorophyll-a and Sea Surface Temperature Images With a New Validation Index
}

\author{
Zineb El Abidi ${ }^{\circledR}$, Khalid Minaoui ${ }^{\circledR}$, Member, IEEE, Anass El Aouni ${ }^{\circledR}$, \\ Ayoub Tamim ${ }^{(}$, and Hicham Laanaya
}

\begin{abstract}
This research deals with the problem of identifying and extracting effectively the main Moroccan upwelling front. The proposed methodology, based on the image-fusion concept, comes to benefit from the information available in both sea-surface temperature (SST) and chlorophyll-a satellite images. Moreover, a new validation index is proposed by computing a simple gradient along the extracted upwelling limit. The developed procedure is applied over a database of 366 SST and 366 chlorophyll-a images from 2007 to 2014, covering the Moroccan Atlantic coast. The final results are validated qualitatively by an oceanographer and quantitatively by our innovative index. The findings of validation demonstrate the performance of our fusion approach.
\end{abstract}

Index Terms-Chlorophyll-a, image fusion, main Moroccan upwelling front, sea-surface temperature (SST), validation index.

\section{INTRODUCTION}

$\mathbf{I}$ NSHORE along the Moroccan Atlantic coast presents one of the most productive ecosystems in the world. It is characterized by the presence of the coastal upwelling phenomenon almost all year round. The hydrodynamical process of the upwelling along this region is mostly forced by the trade winds that cause cold, nutrient-rich intermediate waters to surface at the epipelagic level [1]. As a result, a variable cold and nutrient-rich current operates the coast from North to South.

Such a phenomenon can be identified from the remotely acquired images of the sea-surface temperature (SST). Such images provide a synoptic view of the temperature distribution of the skin (first micrometers of the surface layer) of the surface ocean. This phenomenon can also be identified based

Manuscript received November 12, 2019; revised May 4, 2020; accepted June 2, 2020. This work was supported by Centre National pour la Recherche Scientifique et Technique (CNRST) through the Research Excellence Awards Program and by the "Projets Prioritaires de Recherche under Project PPR2-6 porté par Khalid Minaoui”. (Corresponding author: Zineb El Abidi.)

Zineb El Abidi, Khalid Minaoui, and Hicham Laanaya are with the LRIT Laboratory, CNRST (URAC29), IT Rabat Center, Faculty of Sciences, Mohammed V University in Rabat, Rabat 10056, Morocco (e-mail: zineb.elabidi@um5s.net.ma,khalid.minaoui@um5.ac.ma).

Anass El Aouni is with the Airsea Team, Inria Grenoble Alpes and Laboratoire Jean Kuntzmann, 38000 Grenoble, France.

Ayoub Tamim is with the Department of Marine Fisheries, Higher Institute of Marine Fisheries (ISPM), Agadir 80000, Morocco.

Color versions of one or more of the figures in this letter are available online at http://ieeexplore.ieee.org.

Digital Object Identifier 10.1109/LGRS.2020.3002473 on the satellite images of the ocean color. Unlike the SST data (which comes directly from the ocean's proper radiation in the infrared or in the microwave), ocean color is determined by the interactions between the visible solar radiation and the constituents that are in suspension in sea water. Phytoplankton and organic matter have their proper spectral characteristics, and ocean color data can be calibrated to provide data sets of chlorophyll-a concentration (Chl-a) in proper units $\left(\mathrm{mg} / \mathrm{m}^{3}\right)$. Chlorophyll-a concentration data provide an estimate of the living phytoplankton biomass over the optical depth in the surface layer (20 $\mathrm{m}$ in the open ocean)

Several authors have used both satellite images SST and chlorophyll-a concentration to characterize different features of the Moroccan upwelling and other systems. For instance, El Aouni et al. [2], [4], and Rossi et al. [3], [5] studied the nonlinear relationship between the physical process in the upwelling areas and their impacts on the biological productivity.

Some studies focused on studying the variability of this phenomenon using different indices. Benazzouz et al. [6] proposed a thermal-based index that was used to characterize the main upwelling pattern in the region. This index was improved by El Aouni et al. [7] and was used to study the upwelling seasonality of the region. Chl-a images were also used to compute the upwelling indices, and Demarcq et al. [8] defined an upwelling index from its biological side as the integration of the Chl-a concentration from the coastline to the limit of the extracted upwelling contour.

Other studies focused on the problem of the upwelling identification from the SST and Chl-a images. Tamim et al. [9]-[12] proposed a clustering-based approach to identify the upwelling areas from the SST images. Recently, El Aouni et al. [7] improved the earlier method by introducing a simple normalization to allow these methods to work properly on the other part of the system. In a related work, El Abidi and Minaoui [13] and El Aouni et al. [14] proposed different methods to identify the upwelling along the Atlantic coast of Morocco based on the biological observation. El Aouni et al. [1] and El Abidi et al. [15] developed different methodologies to delineate and extract the upwelling region using the satellite observation. In fact, 
the method proposed in [1] was used to study the upwelling physical and biological variabilities of the northwest African region.

In this letter, we add to the literature of this particular region of the world a new study that combines both biological and physical satellite images. The method developed in this letter defines the upwelling regions based on segmenting both images and combining their output by using a fusion process. A main reason behind this choice is to provide an operation that ensures the continuity of the daily outputs for a better monitoring. A simple example is having cloud pixels in different areas in the SST and chlorophyll-a, and our proposed method will combine the outputs of both observations into one single output, which will ensure the spatial continuity of the phenomenon and, therefore, a better monitoring. Another reason behind this is the physics of the phenomenon that we want to detect the areas that are characterized by both cold and nutrient water, which is exactly what defines the upwelling water. By that, we avoid several data deficiencies such as dust that might affect the temperature pixels in the western Sahara harmful pigment that can be detected in the chlorophyll-a images. Moreover, we propose a new index to validate our method.

The current letter is organized as follows. In Section II, we define the data sets and the studied area. Section III describes the proposed method that improves the detection of the upwelling regions. The validation of the results is discussed in Section IV before we conclude in Section V.

\section{Data And ARea Studied}

The database used in this letter is made available by the NASA's ocean color Website http://oceancolor.gsfc.nasa.gov/. The region of interest spans from $21^{\circ} \mathrm{N}$ to $36^{\circ} \mathrm{N}$ and $6^{\circ} \mathrm{E}$ to $19^{\circ} \mathrm{E}$. Each satellite image is obtained weekly by the MODIS sensor that is embedded on the NASA's Terra satellite. It is represented by a $378 \times 378$ pixel map with a spatial resolution of $4 \times 4 \mathrm{~km}$. In keeping with our fusion approach, the database contains two types of remotely sensed image. The first is the Chl-a image expressed in milligrams of chlorophyll per-cubic meter of seawater and the second is the SST expressed in Celsius degrees.

A color scale was applied to each image in order to get the best distribution of the color levels over the Chl-a and SST range in each image individually. The white color is reserved for land and clouds that are represented by the NaN's value.

A total of $366 \mathrm{Chl}-\mathrm{a}$ and 366 SST images are processed to cover all the Moroccan coast during the period of 2007-2014. The example treated in this letter is shown in Fig. 1.

\section{Proposed Methodology}

The purpose of this letter is to introduce an upwelling fusion approach that improves the extraction and delimitation of the coastal upwelling areas using both SST and Chl-a images. The proposed approach consists of the four following phases.

\section{A. Preprocessing by Polynomial Curve Fitting}

To produce high quality for upwelling extraction, we required preprocessing for fixing the cloudiness problem

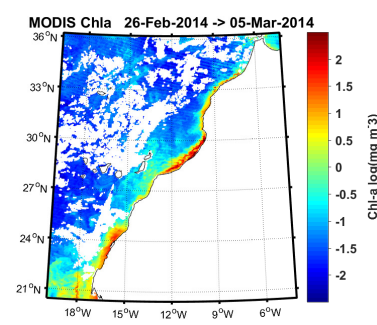

(a)

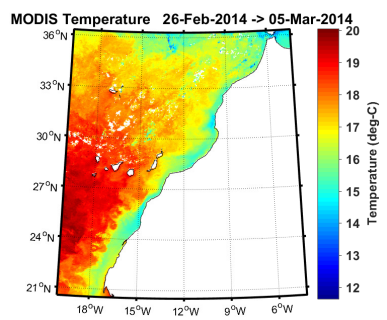

(b)
Fig. 1. Chosen example images. (a) Chlorophyll-a concentration image chosen from our database. (b) SSTI.

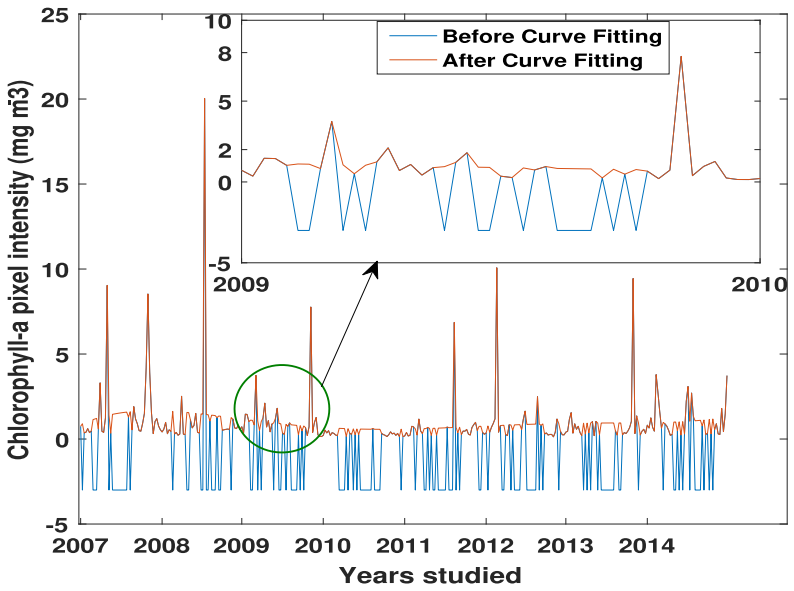

Fig. 2. Chlorophyll pixel intensity variation before and after curve fitting in all the databases.

prior to the fusion process. Depending on the database exploited, the Chl-a images are the most cloudiness data compared with what is present in the thermal observations. This is because of the different wavelengths and algorithms used to provide such data. In fact, the Moroccan Atlantic waters can be under partial or complete cloud covers, as shown in the left of Fig. 1(a). The polynomial curve fitting [16] method was implemented here in order to solve the cloud-pixel issue together with filling these pixels without modifying the unclouded ones. Its mathematical expression is represented in the following equation:

$$
\begin{aligned}
\mathcal{P}_{n}(X)=p_{0}+p_{1} \times X^{1} & +p_{2} \times X^{2} \\
& +\cdots+p_{n-1} \times X^{n-1}+p_{n} \times X^{n} .
\end{aligned}
$$

The coefficients in $\mathcal{P}_{n}$ are in ascending powers, where the value of the polynomial degree is $n$. In our case, the value of the degree is $5(n=5)$, because it is the best fit (in a least-squares sense) for the original chlorophyll images and also because no change will be made in the pixel-intensity variation.

The curve in Fig. 2 displays the intensity variation of an example of one pixel with coordinates $P(175,215)$ existed in cold waters and in high chlorophyll-a concentration region. As shown, the negative noisy values generated by the clouds are rectified finely by the polynomial curve fitting in each sensed Chl-a image of the whole database used. Indeed, to validate the results of this first step, we adopted the 


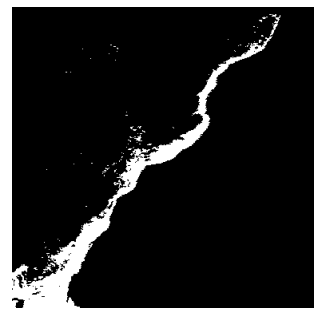

(a)

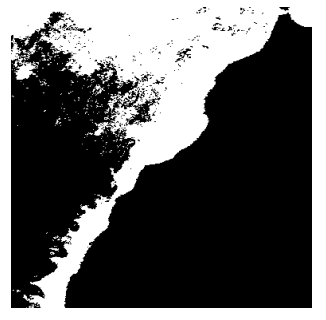

(b)

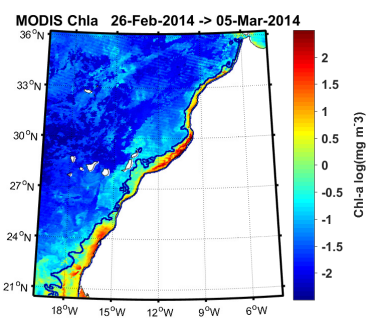

(a)

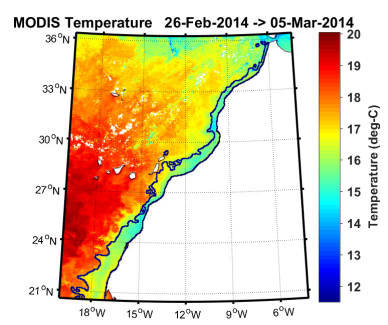

(b)
Fig. 3. FCM results on the chosen example images. (a) Classified chlorophyll image (Chl-aI). (b) Classified SSTI.

universal image quality index "Mean squared error." Effectively, we found that the mean of the MSE in all the processed images is $0.21(\mathrm{MSE}=0.21)$.

\section{B. Clustering by Fuzzy C-Means}

The second step of the proposed methodology seeks to define upwelling waters by applying the fuzzy C-means (FCM) method. FCM is an unsupervised technique based on the fuzzy logic clustering [17], and it is frequently implemented in the pattern-recognition field. This method allows each data point to be in multiple clusters with different probabilities. Mathematically, FCM is the minimization solution of the following equation:

$$
J(X ; U, v)=\sum_{i=1}^{C} \sum_{k=1}^{N}\left(\mu_{i k}\right)^{m}\left\|x_{k}-v_{i}\right\|^{2}
$$

where $X=\left[x_{1}, x_{2}, x_{3}, \ldots, x_{N}\right]$ is a vector of the analyzed image (Chl-a or SST) intensity at the $k$ th pixel. The parameters $C$ and $v$ are, respectively, the number of clusters and cluster centroids, while $U=\left[\mu_{i j}\right]$ represents the fuzzy partition matrix and $\left\|x_{k}-v_{i}\right\|$ the Euclidean norm. Criterion $m$ is a weighting exponent that controls the fuzziness of the membership of each point. It is constrained by $m>1$. The problem of nonlinear optimization in (2) is fixed by using jointly the below membership functions and cluster centroids

$$
\begin{aligned}
\mu_{i k} & =\frac{1}{\sum_{j=1}^{C}\left(\frac{\left\|x_{k}-v_{i}\right\|}{\left\|x_{k}-v_{j}\right\|}\right)^{\left(\frac{2}{m-1}\right)}}, 1 \leq i \leq C, \quad 1 \leq k \leq N \\
v_{i} & =\frac{\sum_{j=1}^{N}\left(\mu_{i j}\right)^{m} x_{j}}{\sum_{j=1}^{N}\left(\mu_{i j}\right)^{m}} .
\end{aligned}
$$

Finding the optimal number of clusters is a major difficult part of FCM implementation. In the actual letter, the determination of this number is based on [9] to localize the cold waters in SST observation and on [18] to define the nutrient-rich region in the Chl-a satellite image. As result of that, the number of clusters is $2(C=2)$ with regard to process later a binary image comporting just two classes: Upwelling and not-upwelling.

Fig. 3 presents the outputs of the FCM clustering technique applied in 1. In fact, the white pixels refer to the desired upwelling area, whereas the black pixels correspond to offshore waters, none belonging to our region of interest. When the northern offshore temperatures are much lower and/or very
Fig. 4. Result of fusion marked by canny colored filter superimposed (a) on the corrected Chl-a image and (b) on SST image, respectively.

close to the southern upwelling temperatures, FCM (as a linear clustering method) considers the northern part (from $28^{\circ} \mathrm{N}$ to $36^{\circ} \mathrm{N}$ ) as the upwelling zone, while it is not that generates the undersegmentation problem. To clear up this issue, we process the below stage.

\section{Fusion by Logical AND Operator}

In general, the efficient way to improve the detection of an object in image processing is fusion. In fact, this term is defined as the combination of two or more images into a new fused image that is more informative, ensuring a better quality than the input ones [19]. In this sense, the inputs of the actual stage are the output of the FCM over the SST and Chl-a data. Effectively, the logical AND operator is applied, it returns True if both operands [Chl-aI and sea surface temperature image (SSTI) labeled images] had the information of upwelling existence, and it gives False otherwise (lack of cold, nutrient-rich waters).

\section{Postprocessing by Mathematical Morphology}

After determining the zone covered by the upwelling waters, the resting problem concerns the deletion of some tiny and noisy structures in the offshore not belonging to the upwelling region. A mathematical morphology technique is implemented here to remove these residual pixels using the opening operation. This latter is started by the erosion of all connected components that are fewer than ten pixels without eliminating the unconnected pixels existed near the coastline.

The result of all the steps is illustrated in Fig. 4, where the upwelling boundary is marked in bolder line on the rectified Chl-a image [Fig. 4(a)] and on the chosen SST observation [Fig. 4(b)]. The results show that the proposed methodology seems to be pertinent to upwelling identification.

\section{RESUlts AND VALIDATION}

\section{A. Qualitative Validation}

Due to the difficulty in validating visually pixel by pixel this gigantic database, the oceanographer took the challenge to validate 92 images for two successive years (2013 and 2014) based on his knowledge on the spatiotemporal variation of the Moroccan upwelling. Corresponding to the validation notation stated in [20], "Poor" was assigned when the proper main upwelling front is not detected; in this case, the proposed methodology might overestimate by shrinking (oversegmentation) or expanding (undersegmentation) the size of the 


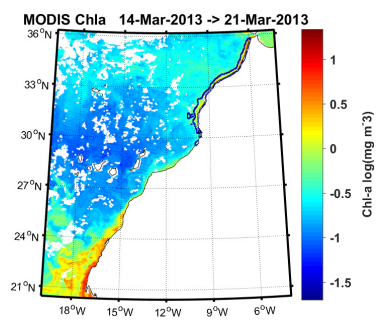

(a)

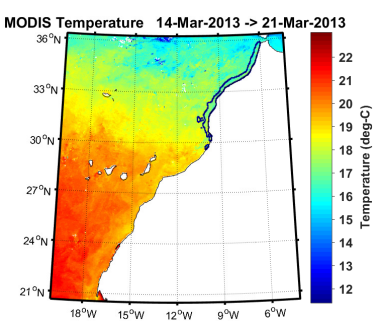

(b)
Fig. 5. Example of the grade "Poor" of the oceanographer validation superposed on the original (a) chlorophyll and (b) SST images.

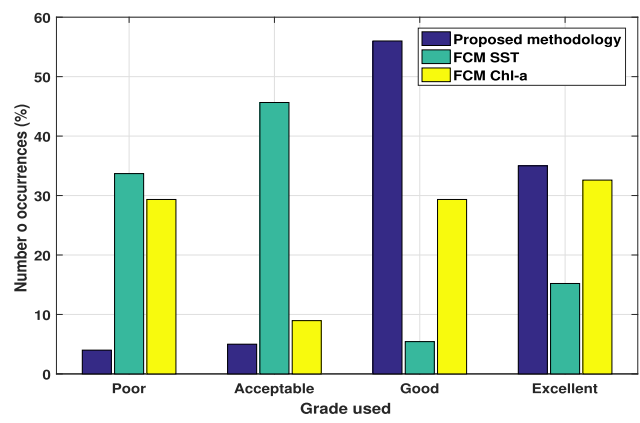

Fig. 6. Qualitative interpretation made by the oceanographer on the fusion of 92 Chl-a and 92 SST images of 2013 and 2014.

upwelling area and/or where the clouds cover up principally the Coastline (this grade is noted by the oceanographer from 1 to 4 per 10 and it is attributed to Fig. 5), while "Excellent" was assigned when the extracted limit is exactly the main upwelling front (this range is noted from 9 to 10 and it is attributed to Fig. 4).

According to the assessment presented in Fig. 6, we can see that the grade "Poor" reached $4 \%$ for our methodology, and $33 \%$ and 29\%, respectively, for the results of FCM over SST and Chl-a. Overall, the two grades "Good" and "Excellent" are making a total of $91 \%$ for the proposed methodology and $62 \%$ is reached for the results of FCM on Chl-a and only $21 \%$ for the FCM SST results. After evaluating qualitatively the three methods over this representative database, we can conclude that the results of FCM on the SST observations are the worst results for detecting the upwelling waters because of the problem of undersegmentation. Comparing our methodology with the FCM Chl-a results, we remark that the values of "Excellent" grade are nearly similar; however, the current method (color blue) stays the most significant approach at the hand of detecting the region of interest in all the validated database.

By considering the qualitative evaluation of our previous works provided in [20] and [21], we can affirm that the proposed methodology is more proficient for delineating the Moroccan coastal upwelling areas.

\section{B. Quantitative Validation}

The qualitative validation is subjective and needs a domain expert to validate the results. Accordingly, to demonstrate the performance of our method, we examine objectively the results by computing a simple gradient along the extracted upwelling limit (difference between the limits of the adjacent pixel in

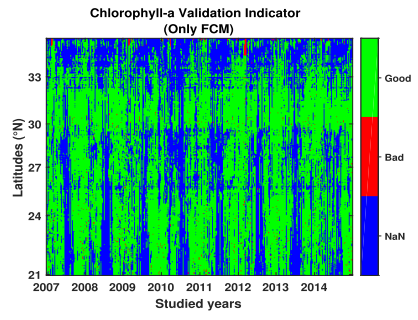

(a)

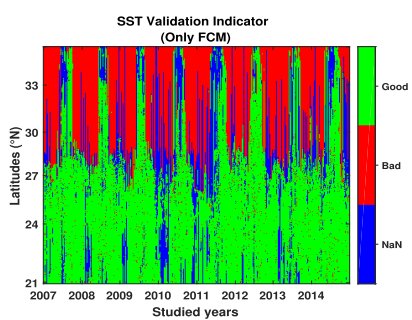

(c)

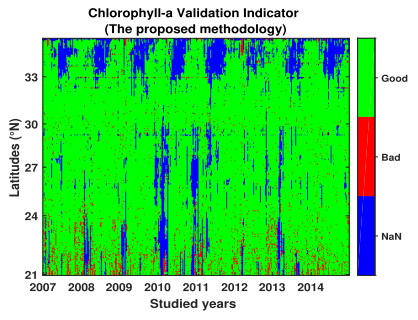

(b)

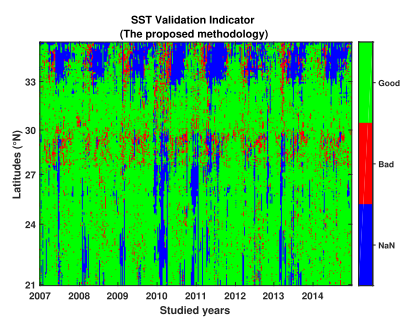

(d)
Fig. 7. Time series during the entire study period, highlighting the validation indicator for the Chl-a and SST data using FCM versus our methodology. (a) and (c) Result of validation indicator respectively over Chl-a and SST data using only FCM, while, the right illustrations are the finding of this indicator using the proposed methodology over 366 chlorophyll-a, (b) 366 SST, and (d) images.

the offshore and the other in the upwelling region). Pixels belonging to the upwelling zone are cold and characterized by strong chlorophyll concentration compared with the ones in the offshore water. Therefore, a good upwelling detection should present a positive (negative) gradient over the SST (Chl-a) images, while $\mathrm{NaN}$ is attributed when there is no upwelling limits on the specified latitude.

Fig. 7 highlights the results of the validation indicator of FCM over the Chl-a and SST images [left range of Fig. 7(a)-(c)] and of our fusion approach (right range). Regarding Fig. 7(b), our method grants largely the correction of cloud pixels in all the Moroccan latitudes during the period from 2007 to 2014, whereas it improves the detection together with solving markedly the undersegmentation problem, as illustrated in Fig. 7(d).

The computed plots provide us the calculation of a new validation upwelling index $\left(V_{\mathrm{Up}}\right)$. We define our original $V_{\mathrm{Up}}$ as follows:

$$
V_{\mathrm{Up}}=\frac{\text { Number }_{\mathrm{NP}}}{L_{I} \times \text { Number }_{I}}
$$

where Number $_{\mathrm{NP}}$ is the number of well-classified latitude steps from each type Chl-a and SST image. In fact, we determine it as the sum of good values along all the studied years. Indeed, $L_{I}$ in (5) is considered as the number of latitude steps over the Moroccan Atlantic coast area, while Number $_{I}$ is the total number of processed images (366). The results of $V_{\mathrm{Up}}$ are shown in Table I, and the wide difference between the obtained values without and with the proposed methodology tested on each data type are noted.

The outputs of this index confirm that our approach operates largely the delimitation of the true limits corresponding to the main Moroccan upwelling fronts with a percentage higher than $20 \%$ for the Chl-a in comparison with the $V_{\mathrm{Up}}$ of the FCM 
TABLE I

RESUlts of the VALIDATION UPWELLING INDEX ( $\left.V_{\text {UP }}\right)$ ON ChL-A AND SST Data Without/With the Proposed Methodology

\begin{tabular}{|l|l|l|}
\cline { 2 - 3 } \multicolumn{1}{c|}{} & FCM only & The proposed methodology \\
\hline$V_{U p}$ on Chl-a & 0.625 & 0.826 \\
\hline$V_{U p}$ on SST & 0.542 & 0.759 \\
\hline
\end{tabular}

only and more than $21 \%$ for the SST database in the eight years. Furthermore, the proposed procedure beats markedly the undersegmentation problem shown in the north of the binary SST images. To sum up, the findings of the quantitative validation show clearly the performance of the suggested fusion methodology. Altogether, this validation supports broadly the qualitative interpretation made by the oceanographer.

\section{CONCLUSION}

This letter exposed a fusion process of the Chl-a and SST remotely sensed images in favor of detecting automatically and efficiently the main upwelling front, separating the cold waters and the warm offshore waters. The procedure started by a preprocessing step in order to rectify the noisy cloud pixels located mostly in the Chl-a data. After that, we apply FCM, which is an unsupervised classification method on the two kinds of our database; then, we employed the logical AND operator in the interest of enhancing the results and also to overcome the problem of undersegmentation represented in the classified SST image. In the last step, we used a morphological technique in order to remove the residual pixels not belonging to the upwelling areas. The proposed methodology is validated qualitatively by an oceanographer and objectively by our innovative index over eight years. Both of these evaluations have demonstrated that our model performance is better than using an SST image or a Chl-a image individually.

\section{REFERENCES}

[1] A. El Aouni, V. Garcon, J. Sudre, H. Yahia, K. Daoudi, and K. Minaoui, "Physical and biological satellite observations of the Northwest African upwelling: Spatial extent and dynamics," IEEE Trans. Geosci. Remote Sens., vol. 58, no. 2, pp. 1409-1421, Feb. 2020.

[2] A. El Aouni, K. Daoudi, H. Yahia, K. Minaoui, and A. Benazzouz, "Surface mixing and biological activity in the North-West African upwelling," Chaos, Interdiscipl. J. Nonlinear Sci., vol. 29, no. 1, Jan. 2019, Art. no. 011104.

[3] V. Rossi, C. López, E. Hernández-García, J. Sudre, V. Garçon, and Y. Morel, "Surface mixing and biological activity in the four Eastern Boundary upwelling systems," 2009, arXiv:0909.0115. [Online]. Available: http://arxiv.org/abs/0909.0115

[4] A. El Aouni, K. Daoudi, H. Yahia, and K. Minaoui, "Surface mixing and biological activity in the North African upwelling," AGU Ocean Sci. Meetings, Portland, OR, USA, Tech. Rep., 2018.
[5] V. Rossi, C. López, J. Sudre, E. Hernández-García, and V. Garçon, "Comparative study of mixing and biological activity of the Benguela and Canary upwelling systems," Geophys. Res. Lett., vol. 35, no. 11, pp. 1-5, 2008.

[6] A. Benazzouz et al., "An improved coastal upwelling index from sea surface temperature using satellite-based approach-The case of the Canary Current upwelling system," Continental Shelf Res., vol. 81, pp. 38-54, Jun. 2014.

[7] A. El Aouni, K. Daoudi, K. Minaoui, and H. Yahia, "Robust detection of the North-West African upwelling from SST images," IEEE Geosci. Remote Sens. Lett., early access, Apr. 9, 2020, doi: 10.1109/LGRS.2020.2983826.

[8] H. Demarcq, R. Barlow, and L. Hutchings, "Application of a chlorophyll index derived from satellite data to investigate the variability of phytoplankton in the Benguela ecosystem," Afr. J. Mar. Sci., vol. 29, no. 2, pp. 271-282, Aug. 2007.

[9] A. Tamim, K. Minaoui, K. Daoudi, H. Yahia, A. Atillah, and D. Aboutajdine, "An efficient tool for automatic delimitation of Moroccan coastal upwelling using SST images," IEEE Geosci. Remote Sens. Lett., vol. 12, no. 4, pp. 875-879, Apr. 2015.

[10] A. Tamim et al., "A simple and efficient approach for coarse segmentation of Moroccan coastal upwelling," in Proc. 21st Eur. Signal Process. Conf. (EUSIPCO), 2013, pp. 1-5.

[11] A. Tamim et al., "A simple tool for automatic extraction of Moroccan coastal upwelling from sea surface temperature images," in Proc. 9th Int. Conf. Intell. Syst., Theories Appl. (SITA), May 2014, pp. 1-4.

[12] A. Tamim, K. Minaoui, K. Daoudi, A. Atillah, and D. Aboutajdine, "On detectability of Moroccan coastal upwelling in sea surface temperature satellite images," in Proc. Int. Symp. Vis. Comput. Cham, Switzerland: Springer, 2014, pp. 386-395.

[13] E. A. Zineb and M. Khalid, "A simple tool for automatic extraction of the Moroccan coastal upwelling from sea surface chlorophyll images," in Proc. Intell. Syst. Comput. Vis. (ISCV), Apr. 2017, pp. 1-4.

[14] A. El Aouni, K. Minaoui, A. Tamim, K. Daoudi, and H. Yahia, "An improved method for accurate computation of coastal upwelling index using sea surface temperature images," in Proc. 9th Int. Symp. Signal, Image, Video Commun. (ISIVC), Nov. 2018, pp. 76-81.

[15] Z. El Abidi, K. Minaoui, A. Tamim, and H. Laanaya, "Delineation of Moroccan coastal upwelling using the principal component analysis fusion algorithm on SSC and SST images," in Proc. 9th Int. Symp. Signal, Image, Video Commun. (ISIVC), Nov. 2018, pp. 174-178.

[16] G. T. Thompson and S. J. Balch, "An efficient algorithm for polynomial curve fitting," Comput. Geosci., vol. 14, no. 5, pp. 547-556, Jan. 1988.

[17] J. C. Bezdek, R. Ehrlich, and W. Full, "FCM: The fuzzy C-means clustering algorithm," Comput. Geosci., vol. 10, nos. 2-3, pp. 191-203, Jan. 1984

[18] A. El Aouni et al., "Detection of Moroccan coastal upwelling using sea surface chlorophyll concentration," in Proc. IEEE/ACS 12th Int. Conf. Comput. Syst. Appl. (AICCSA), Nov. 2015, pp. 1-4.

[19] I. Bloch and H. Maitre, "Data fusion in 2D and 3D image processing: An overview," in Proc. 10th Brazilian Symp. Comput. Graph. Image Process., 1997, pp. 127-134.

[20] Z. Elabidi, K. Minaoui, A. Tamim, and H. Laanaya, "A simple fusion approach of chlorophyll images and sea surface temperature images for improving the detection of Moroccan coastal upwelling," in Proc. IEEE Int. Geosci. Remote Sens. Symp. (IGARSS), Jul. 2018, pp. 7208-7211.

[21] Z. El Abidi, K. Minaoui, A. Tamim, and H. Laanaya, "Detection of Moroccan coastal upwelling using the alpha blending fusion technique of sea surface chlorophyll images and sea surface temperature images," in Proc. 4th Int. Conf. Adv. Technol. Signal Image Process. (ATSIP), Mar. 2018, pp. 1-5. 